\title{
A Streamlined Strategy for Aglycone Assembly and Glycosylation
}

\author{
Dr. Katherine M. Partridge, Dr. Scott J. Bader, Dr. Zachary A. Buchan, Dr. Christopher E. \\ Taylor, and Prof. John Montgomery \\ Department of Chemistry, University of Michigan, Ann Arbor, MI 48109-1055, Fax: \\ (+1)734-763-1106, Homepage ((optional)): http://www.umich.edu/ jmgroup
}

John Montgomery: jmontg@umich.edu

\section{Abstract}

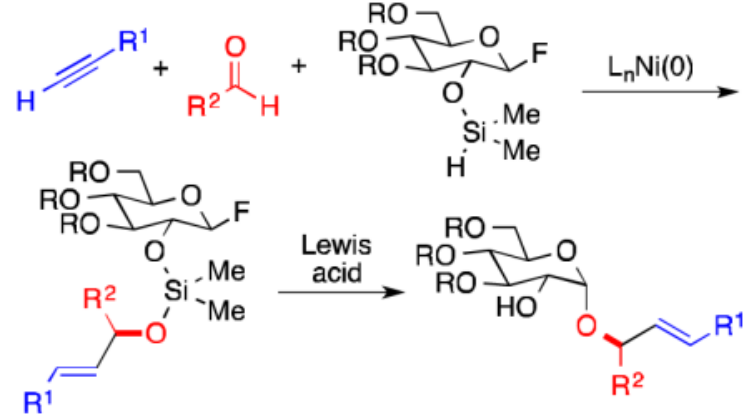

Multipurpose sugar silanes-Carbohydrate-derived silane reagents are utilized as the reductant for nickel-catalyzed aldehyde-alkyne reductive couplings, followed immediately by intramolecular glycosylation of the newly formed protected hydroxyl The approach, illustrated in the catalyzed conversion of aldehydes and alkynes to glycosylated allylic alcohols, enables the assembly of the carbon-carbon framework and stereochemical features of an aglycone while simultaneously establishing the site of glycosylation.

\section{Keywords}

glycosylation; nickel; coupling; multicomponent; silicon-tether

The assembly of glycosylated structures, even with relatively simple aglycones, often presents a significant challenge in chemical synthesis. ${ }^{[1]}$ Much of this complexity derives from the linear approach required by existing synthetic strategies. A typical strategy involves initial assembly of the aglycone by a collection of stereoselective C-C bondforming processes. During the sequence, orthogonal protecting groups are installed, and at a late stage, a single hydroxyl is then unmasked for a glycosylation event at the end of the synthesis. We envision that an alternate, more efficient approach might allow a $\mathrm{C}-\mathrm{C}$ bondforming step to be merged with a glycosylation event. Since carbonyl addition reactions, which are routine in many aglycone synthesis strategies, inherently generate an alcohol functionality, the direct capture of the forming hydroxyl in a glycosylation event could provide an alternate strategy with advantages in efficiency. Towards this end, our laboratory has devised a new class of reagents 1 that possesses a carbohydrate core with the anomeric

Correspondence to: John Montgomery, jmontg@umich.edu.

Supporting information for this article is available on the WWW under http://www.angewandte.org or from the author. 
position functionalized for glycosylation and with the $\mathrm{C} 2$ hydroxyl protected as a reactive silicon hydride (Scheme 1). ${ }^{[2]}$ These multifunctional sugar silane reagents thus possess a silyl hydride that could enable a variety of catalytic reductive processes including C-C bond formations, while the anomeric position is derivatized to directly enable glycosylation.

The appeal of such reagents derives from the broad array of transition metal-catalyzed processes that are enabled by the reactivity of silicon hydrides, ${ }^{[3]}$ coupled with the considerable utility of silicon-tethered intramolecular glycosylations demonstrated by Stork and Bols. ${ }^{[4]}$ Sugar silane reagents were previously utilized in a ketone hydrosilylation/ intramolecular glycosylation sequence to provide site-selective reductive glycosylations of simple ketones. ${ }^{[2]}$ However, their use in a process wherein the silane mediates formation of a new $\mathrm{C}-\mathrm{C}$ bond or where a multicomponent coupling process occurs is without precedent. Herein, we illustrate that sugar silanes can mediate catalytic carbon-carbon bond-forming reactions followed directly by intramolecular glycosylation. This multicomponent approach thus allows both the aglycone skeletal assembly and the glycoside bond formation to be addressed in a single strategy from simple precursors including sugar silane $\mathbf{1}$ rather than through stepwise operations that conventional procedures require (Scheme 2).

The synthesis of sugar silane reagents is straightforward, requiring initial preparation of glycosyl sulfide or fluoride precursors with all hydroxyls protected aside from the C2 hydroxyl. Protection of the $\mathrm{C} 2$ hydroxyl with commercially available chlorodimethylsilane then affords sugar silane reagents in high yield. Although unstable to chromatography, these reagents may be stored for months and are easily manipulated in air. The reagents are generally robust in catalytic operations, with the silicon-hydride functionality undergoing a range of metal-mediated additions without affecting the anomeric leaving group. Our preliminary explorations involved catalytic additions of thioglycosides. ${ }^{[5]}$ Although aldehyde-alkyne reductive couplings with thioethyl- and thiophenyl-sugar silanes were generally effective, efforts to develop the ensuing intramolecular glycosylations were unsatisfactory. A representative coupling with cyclohexane carboxaldehyde and octyne utilizing glucose-derived thioethyl sugar silane 1a is depicted (Scheme 3), but a variety of glycosylation methods with intermediate 2 utilizing standard protocols such as TMSOTf / Niodosuccinimide methods ${ }^{[6]}$ or radical cation-based methodology $\left[{ }^{[7]}\right.$ were low-yielding. The origin of the complexity in glycosylation is clearly the sensitivity of the allylic alcohol, as mixtures of elimination products were observed, whereas intramolecular glycosylations of structurally related saturated alcohols were straightforward and high yielding. ${ }^{[2]}$

The above example illustrates that the sugar chirality plays no role in the creation of the stereogenic center, as 1:1 mixtures of diastereomers were routinely observed. This outcome was in fact expected since the silane involvement was previously illustrated to occur after a rate-determining oxidative cyclization of a nickel-aldehyde-alkyne $\pi$-complex $\mathbf{3}$ (Scheme 4) ${ }^{[8]} \mathrm{A} \sigma$-bond metathesis of the sugar silane with metallacycle intermediate $\mathbf{4}$ follows the stereochemistry-determining oxidative cyclization that leads to metallacycle formation. This outcome illustrates that ligand control during the oxidative cyclization is a likely requirement for controlling stereochemistry in this multicomponent addition process.

Based on the above limitations in diastereoselectivity of the C-C bond-forming step and yield of the glycosylation, we turned our attention to chiral ligand-based processes using alternative glycosyl donors. The corresponding glycosyl fluorides ${ }^{[9]}$ behaved analogously to the thioethyl glycoside depicted above using IMes as ligand, therefore we explored the use of chiral $N$-heterocyclic carbene ligands in the aldehyde-alkyne addition process. Couplings of cyclohexane carboxyaldehyde and 1-octyne with the sugar silane $\mathbf{1 b}$ derived from tribenzylglucosyl fluoride were examined utilizing the chiral $N$-heterocyclic carbene ligand 5 previously developed in our labs (Scheme 5). ${ }^{[10]}$ As anticipated, sugar chirality played no 
role in stereoinduction, and the diastereomeric ratios for generation of the reductive coupling products $\mathbf{6 a}$ (from the $R, R-\mathbf{5}$ ) and $\mathbf{6} \mathbf{b}$ (from $S, S-\mathbf{5}$ ) were opposite and of very similar magnitude, with diastereoselectivities being governed by ligand chirality. Following procedures previously developed for intermolecular glycosylations of glycosyl fluorides, ${ }^{[11]}$ intramolecular glycosylation of silyl-linked intermediates $\mathbf{6 a}$ and $\mathbf{6} \mathbf{b}$ afforded exclusively the a-gluco stereochemistry of the diastereomeric glycosidic linkages of glycosides $\mathbf{7 a}$ and $\mathbf{7 b}$. The stereochemistry of the aldehyde-alkyne addition step was confirmed by desilylation of 6a followed by Mosher ester analysis of the resulting allylic alcohol. The newly formed aglucoside linkages of $\mathbf{7 a}$ and $\mathbf{7 b}$ were confirmed by characteristic $J$-values of the anomeric protons.

A series of aldehyde-alkyne combinations were explored for this catalytic C-C bond formation / intramolecular glycosylation sequence (Table 1). Products $\mathbf{7 a}-\mathbf{7 j}$ were obtained as single diastereomers by standard column chromatographic separation. In analogy to the above examples (Scheme 5), two additional combinations of simple aliphatic aldehydes and terminal alkynes underwent efficient couplings with comparable degrees of diastereocontrol in the reductive coupling step (Table 1, entries 1 and 2). An internal alkyne underwent the reductive coupling in relatively good yield, although intramolecular glycosylations of allylic alcohols derived from internal alkynes generally proceeded in modest yield as illustrated by this example (Table 1, entry 3 ). Several examples then were conducted to examine the chemoselectivity of the process. The reaction proved to be very selective for the combination of aldehyde-alkyne couplings in the presence of isolated ketones (Table 1, entry 4), unprotected hydroxyls (Table 1, entry 5), silyl ethers (Table 1, entry 6), and esters (Table 1, entry 7). The ability to tolerate simple ketones in the process (entry 4) is notable in that hydrosilylations of ketones with sugar silanes were efficient using closely related catalyst systems in cases where the aldehyde-alkyne combination was not present. ${ }^{2}$ Additionally, the tolerance of an unprotected hydroxyl (entry 5) is notable for both the reductive coupling reaction as well as the glycosylation. The advantages of the kinetic bias of intramolecular aglycone delivery contribute to chemoselectivity of glycosylation in the presence of an unprotected hydroxyl. While silyl deprotection occurred during $\mathrm{Bu}_{4} \mathrm{NF}$ treatment of the crude reaction mixture (entry 6), the directing influence of the silyl tether nonetheless allowed selective glycosylation of the secondary allylic alcohol. While the three-component couplings involving mannose silanes efficiently produced silyl intermediates analogous to $\mathbf{6}$, the intramolecular glycosylations to produce $\beta$-mannosides were low-yielding using the current procedure.

The above examples illustrate that ligand chirality allows either diastereomer to be accessed by controlling the stereochemistry of the aldehyde-alkyne addition step. Similarly, we anticipated that ligand control might also allow access to different constitutional isomers of the aglycone by altering regioselectivity of the addition reaction. Since the terminal alkyne addition processes studied above exclusively favor addition of the unsubstituted alkyne terminus to the aldehyde, we examined an alternate ligand class ( \pm )-DP-IPr (Scheme 6). While only available in racemic form, this ligand has previously been illustrated to be highly selective for reversing regioselectivities of terminal alkyne reductive couplings. ${ }^{[12]}$ As an example of this capability in the glycoside bond-forming sequence, reductive coupling of benzaldehyde with cyclohexylacetylene and sugar silane 1b utilizing ( \pm )-DP-IPr as ligand afforded exclusively the regioisomer of silicon-linked intermediate $\mathbf{6} \mathbf{j}$, where the substituted alkyne terminus had undergone addition to the aldehyde (Scheme 6). By using racemic ligand, a 1:1 mixture of diastereomers was obtained, which was converted to a 1:1 ratio of diastereomers of the expected product $\mathbf{7} \mathbf{j}$ upon treatment to the glycosylation conditions described above. 
In summary, sugar silanes have been utilized as versatile reagents for the synthesis of glycosylated allylic alcohols. The sugar silanes serve as the reducing agent in stereocontrolled catalytic C-C bond formations, and then serve to stereoselectively deliver a glycosidic bond through an intramolecular glycosylation process. The full sequence is stereo- and regioselective and is compatible with numerous functional groups. Use of the multifunctional sugar silanes serves to unify the strategy by which aglycone preparation and ensuing glycosylation may be accomplished. On this basis, we anticipate that complex synthesis strategies and challenging site-selective glycosylations may benefit from approaches of this type.

\section{Experimental Section}

General procedure for three-component coupling of an aldehyde, an alkyne and sugar silane 1b. A solid mixture of $\mathrm{Ni}(\mathrm{COD})_{2}(4.2 \mathrm{mg}, 0.015 \mathrm{mmol}), R, R-\mathbf{5} \cdot \mathrm{HBF}_{4}(12.3 \mathrm{mg}, 0.015$ $\mathrm{mmol})$, and $\mathrm{KO}-t-\mathrm{Bu}(1.7 \mathrm{mg}, 0.015 \mathrm{mmol})$ was dissolved in dry THF $(0.75 \mathrm{~mL})$ at $\mathrm{rt}$ under an inert atmosphere of $\mathrm{N}_{2}$. The solution quickly turned a deep, brick red and was stirred for $30-45 \mathrm{~min}$. The aldehyde $(0.15 \mathrm{mmol}, 1.0$ equiv) was added directly to the catalyst solution via microsyringe. A solution of sugar silane (102 mg, $0.2 \mathrm{mmol}, 1.3$ equiv) and alkyne $(0.15 \mathrm{mmol}, 1.0$ equiv) in THF $(0.75 \mathrm{~mL})$ was added to the catalyst solution over 50 min via a syringe drive. At the end of the syringe drive, a second aliquot of alkyne $(0.15$ mmol, 1.0 equiv) in $0.5 \mathrm{~mL}$ dry THF was added over $80 \mathrm{~min}$. The reaction was stirred until disappearance of aldehyde was clearly observed by TLC or overnight, in the instance of incomplete conversion. The reaction mixture was diluted with an equal volume of hexane and filtered through a short plug of silica gel, which was washed with a mixture of EOtAc/ hexanes. The solution was concentrated by rotary evaporation and the residue was purified via flash chromatography $\left(\mathrm{SiO}_{2}\right)$ to afford the desired product.

\section{Supplementary Material}

Refer to Web version on PubMed Central for supplementary material.

\section{Acknowledgments}

The authors wish to thank the National Institutes of Health (GM-57014) and Thermo Fisher (pilot project grant administered by the University of Michigan Life Sciences Institute) for support of this research.

\section{References}

1. a) Toshima K, Tatsuta K. Chem Rev. 1993; 93:1503-1531.b) Zhu XM, Schmidt RR. Angew Chem Int Ed. 2009; 48:1900-1934.

2. Buchan ZA, Bader SJ, Montgomery J. Angew Chem Int Ed. 2009; 48:4840-4844.

3. a) Montgomery J. Angew Chem Int Ed. 2004; 43:3890-3908.b) Riant O, Mostefai N, Courmarcel J. Synthesis. 2004:2943-2958.c) Ojima I, Clos N, Donovan RJ, Ingallina P. Organometallics. 1990; 9:3127-3133.d) Ojima I, Kogure T. Organometallics. 1982; 1:1390-1399.

4. Stork G, Kim G. J Am Chem Soc. 1992; 114:1087-1088.Stork G, LaClair JJ. J Am Chem Soc. 1996; 118:247-248.Bols M. J Chem Soc Chem Commun. 1992:913-914.Bols M. J Chem Soc Chem Commun. 1993:791-792.Jung KH, Muller M, Schmidt RR. Chem Rev. 2000; 100:44234442. [PubMed: 11749353] Cumpstey I. Carbohydr Res. 2008; 343:1553-1573. [PubMed: 18533138] For pioneering studies using other strategies for intramolecular aglycone delivery: Barresi F, Hindsgaul O. J Am Chem Soc. 1991; 113:9376-9377.Ito Y, Ogawa T. Angew Chem Int Ed. 1994; 33:1765-1767.

5. Codee JDC, Litjens R, van den Bos LJ, Overkleeft HS, van der Marel GA. Chem Soc Rev. 2005; 34:769-782. [PubMed: 16100617] 
6. a) Konradsson P, Udodong UE, Fraserreid B. Tetrahedron Lett. 1990; 31:4313-4316.b) Veeneman GH, Vanleeuwen SH, Vanboom JH. Tetrahedron Lett. 1990; 31:1331-1334.c) Ennis SC, Fairbanks AJ, Tennant-Eyles RJ, Yeates HS. Synlett. 1999:1387-1390.

7. Marra A, Mallet JM, Amatore C, Sinay P. Synlett. 1990:572-574.

8. Baxter RD, Montgomery J. J Am Chem Soc. 2008; 130:9662-9663. [PubMed: 18598032] Liu P, Montgomery J, Houk KN. J Am Chem Soc. 2011; 133:6956-6959. [PubMed: 21506540] For related studies with other reductants: McCarren PR, Liu P, Cheong PHY, Jamison TF, Houk KN. J Am Chem Soc. 2009; 131:6654-6655. [PubMed: 19397371]

9. a) Shimizu M, Togo H, Yokoyama M. Synthesis. 1998:799-822.b) Toshima K. Carbohydr Res. 2000; 327:15-26. [PubMed: 10968674]

10. Chaulagain MR, Sormunen GJ, Montgomery J. J Am Chem Soc. 2007; 129:9568-9569. [PubMed: 17628066]

11. a) Kreuzer M, Thiem J. Carbohydr Res. 1986; 149:347-361.b) Wessel HP. Tetrahedron Lett. 1990; 31:6863-6866.

12. a) Malik HA, Sormunen GJ, Montgomery J. J Am Chem Soc. 2010; 132:6304-6305. [PubMed: 20394367] b) Shareef AR, Sherman DH, Montgomery J. Chem Sci. 2012; 3:892-895. [PubMed: 22737401] 


$$
1(\mathrm{X}=\mathrm{SR} \text { or } \mathrm{F})
$$

Scheme 1.

Sugar Silane Reagents. 


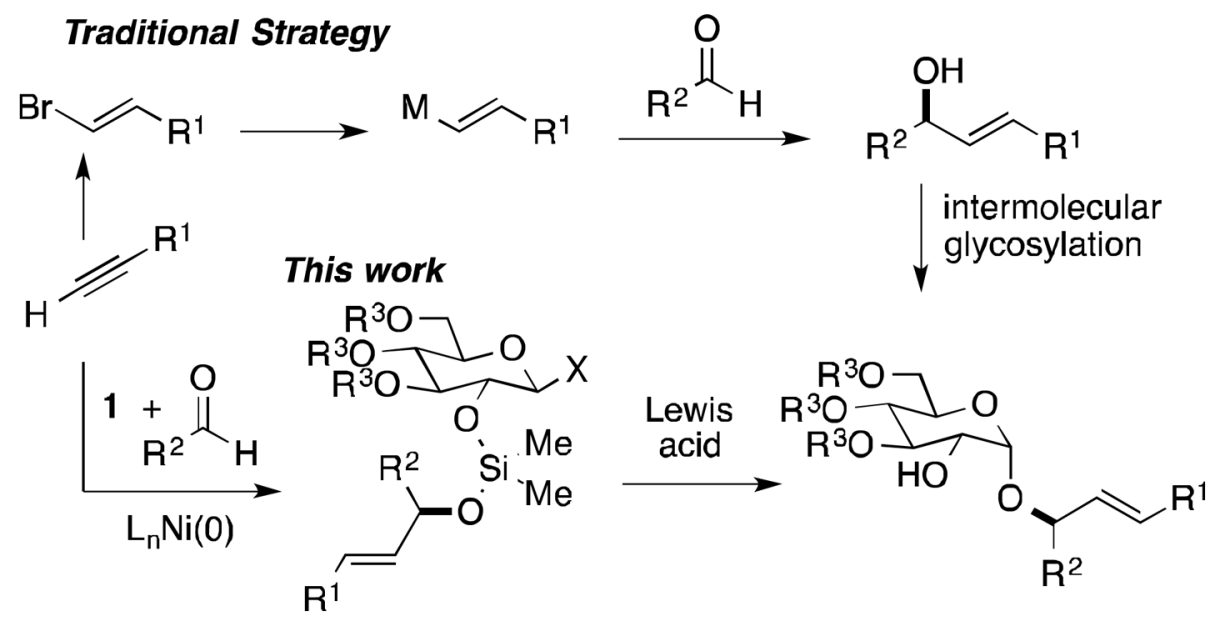

Scheme 2.

New Strategy for Glycoside Synthesis. 


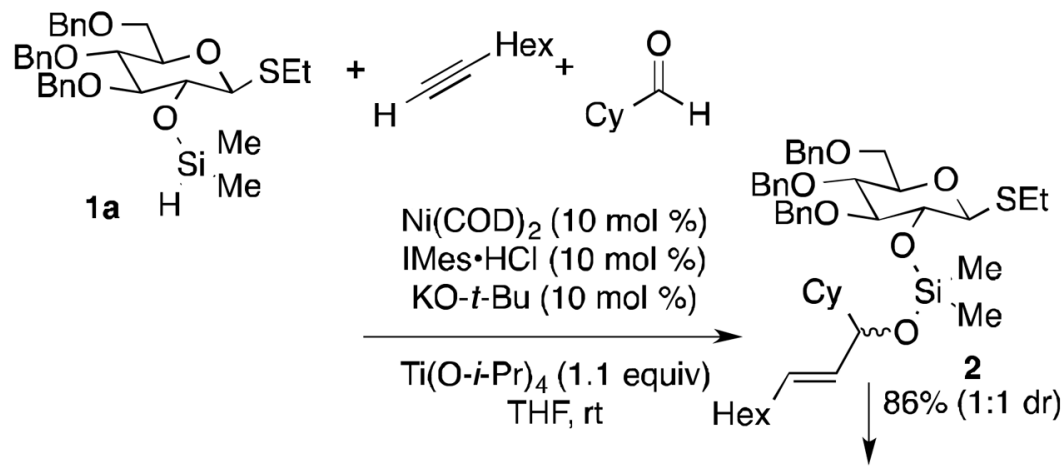

low-yielding intramolecular glycosylation

Scheme 3.

Couplings of Thioglycosides. 


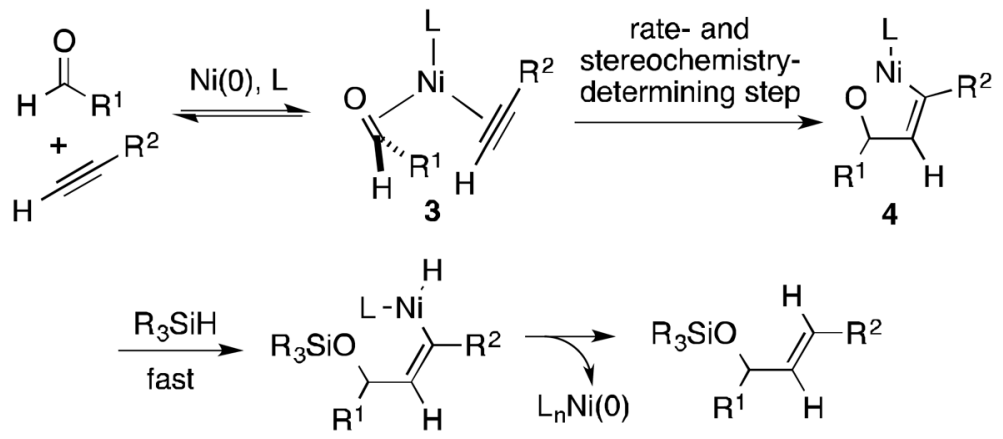

Scheme 4.

Reaction Mechanism. 

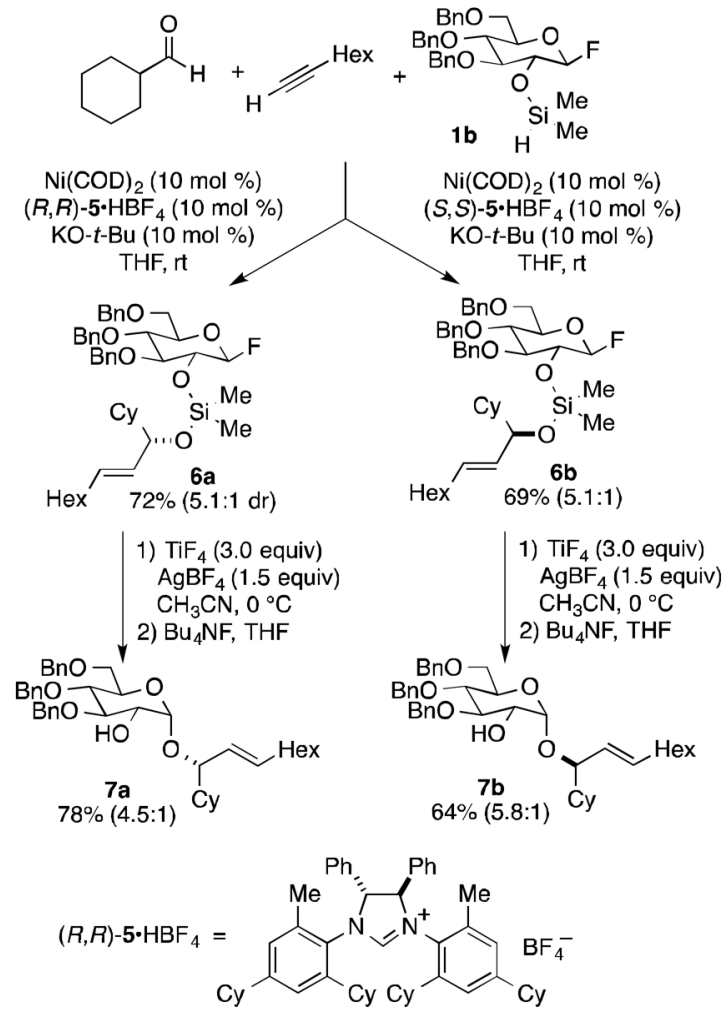

Scheme 5.

Glycosyl Fluoride Reductive Coupling / Glycosylation. 

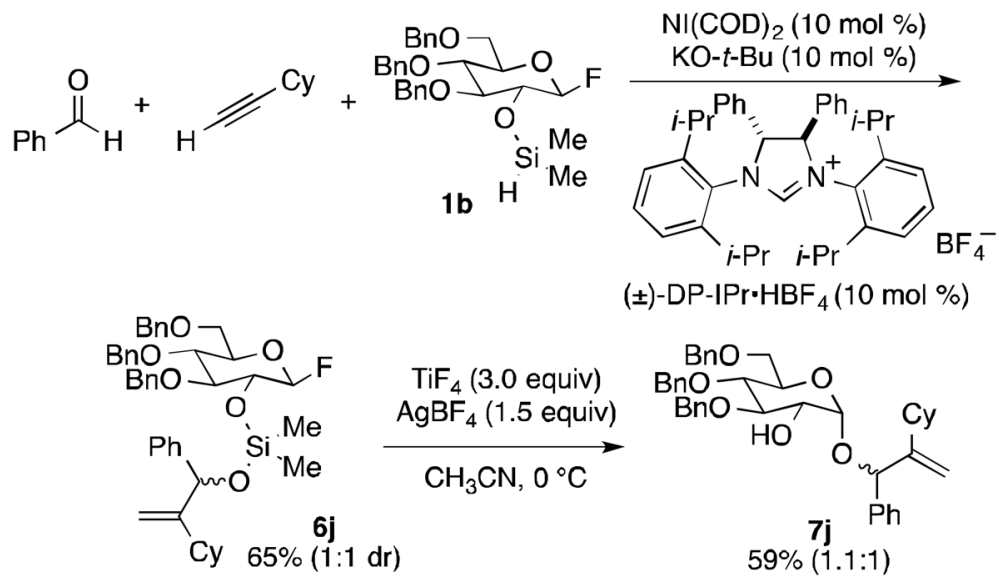

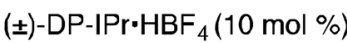

\section{Scheme 6.}

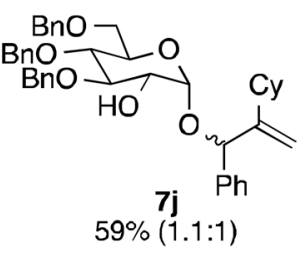

Ligand-Controlled Regiochemistry Reversal. 
Table 1

Scope of Reductive Coupling / Glycosylation.

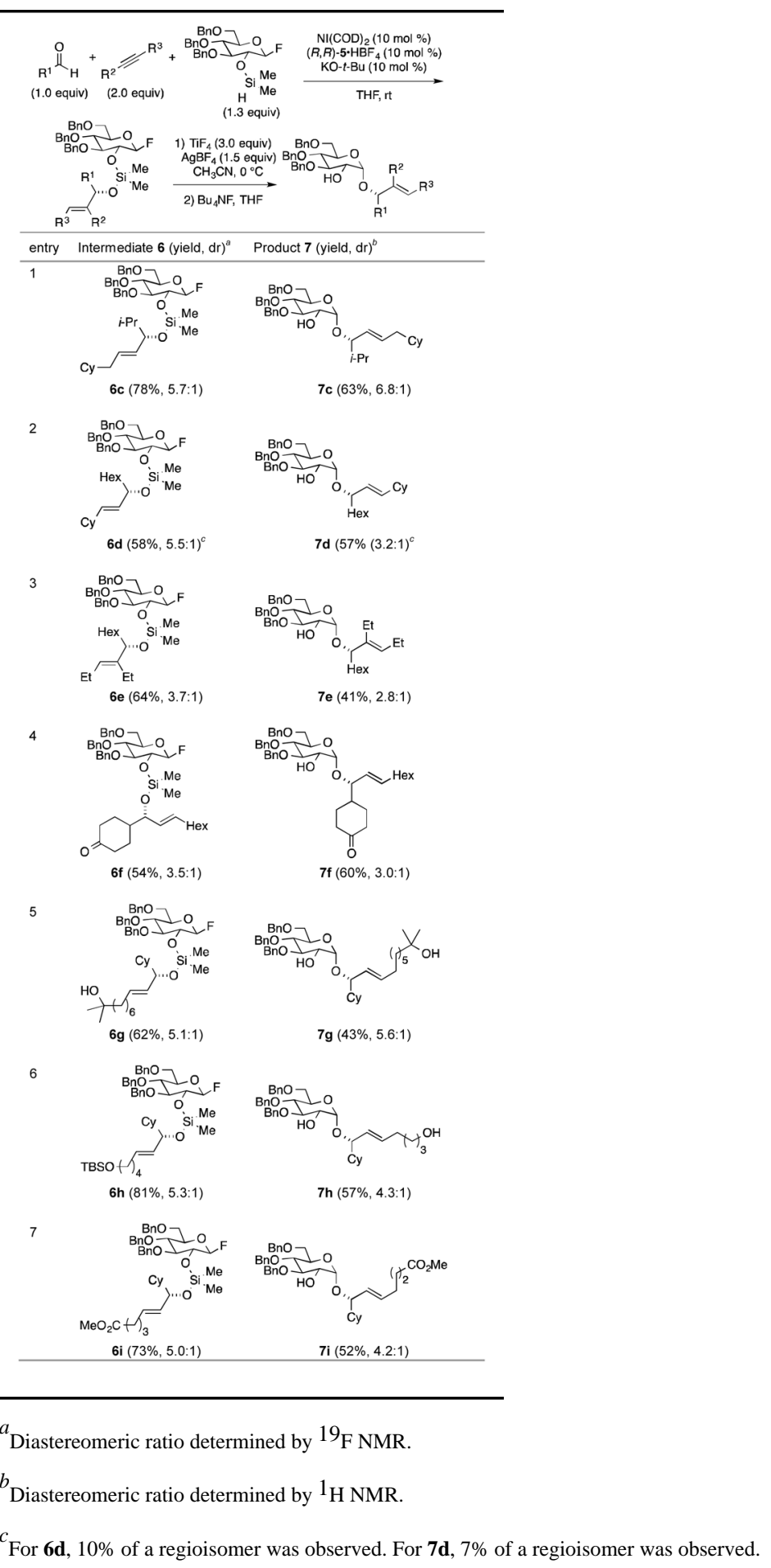

